\title{
Enfermedad animal, zoonosis y 'One health': lo que hemos aprendido los veterinarios a lo largo de la historia
}

\author{
Martí Pumarola Batlle \\ Catedrático de Patología Animal. Departamento de Medicina y Cirugía Animales. Universitat Autònoma de \\ Barcelona (UAB) \\ Subdirector del ICALP \\ ORCID: https://orcid.org/0000-0002-0935-7941
}

Cita recomendada. PUMAROLA BATLLE, M., Enfermedad animal, zoonosis y 'One health': lo que hemos aprendido los veterinarios a lo largo de la historia, dA. Derecho Animal (Forum of Animal Law Studies) 11/4 (2020). - DOI https://doi.org/10.5565/rev/da.520

\section{Resumen}

La pandemia de COVID19 ha puesto en evidencia la transversalidad profesional en el ámbito de la salud, cuando médicos, veterinarios, epidemiólogos, inmunólogos, juristas, sociólogos, ambientalistas, políticos, etc. hemos tenido que colaborar para encontrar y aplicar soluciones al problema. A lo largo de la historia los veterinarios hemos aprendido cómo la salud y la enfermedad de los animales repercutían directa o indirectamente en la población humana. Las zoonosis, enfermedades transmisibles de los animales a los humanos, son un buen ejemplo de ello. Utilizando la historia haremos un recorrido de algunos de estos procesos, para ver cómo los veterinarios han actuado, implicándose y colaborando con otros profesionales, para llegar al concepto actual de una única salud ('One health'). La necesidad de la cooperación multidisciplinar para poder enfrentarnos de forma efectiva a pandemias como la que vivimos en la actualidad resulta fundamental.

Palabras clave: Enfermedad animal; Veterinaria; Zoonosis; Historia.

Abstract - Animal disease, zoonosis and 'One health': what we veterinarians have learned throughout history

The COVID19 pandemic has revealed professional transversality in the field of health, when doctors, veterinarians, epidemiologists, immunologists, jurists, sociologists, environmentalists, politicians, etc. have had to collaborate to find and apply solutions to the problem. Throughout history, veterinarians have learned how animal health and disease directly or indirectly affected the human population. Zoonoses - infectious diseases transmissible from animals to humans - are a good example of this. Using history, we will go through some of these processes to see how veterinarians have acted, involving and collaborating with other professionals, to arrive at the current concept of a single health ('One health'). The need for multidisciplinary cooperation in order to effectively face pandemics such as the one we are currently experiencing becomes fundamental.

Key words: Animal disease; Veterinary; Zoonoses; History. 


\section{Las zoonosis: origen y consecuencias}

Según la Organización Mundial de la Salud (OMS, en inglés WHO $)^{1}$ una zoonosis corresponde a aquella enfermedad y/o infección que se transmita entre humanos y otros animales vertebrados de forma directa y natural. Así tenemos las antropozoonosis, cuando se transmiten de humanos a otros vertebrados, las zooantroponosis, de vertebrados a humanos y las anfixenosis, aquella zoonosis que se transmiten de humanos a vertebrados o viceversa. En los últimos años se han añadido las zoonosis transmitidas por alimentos.

Si nos preguntamos sobre el origen de estas enfermedades zoonóticas, deberíamos limitarnos a la aparición de los primeros homínidos, hace unos pocos millones de años y cómo empezaron a padecerlas y a transmitirlas de un individuo a otro provocando las conocidas como enfermedades epidémicas. Pero si pensamos en los agentes infecciosos que las producen (virus, bacterias, parásitos, hongos etc.) debemos remontarnos mucho tiempo antes. ¿Cuándo aparecieron por primera vez? La respuesta a esta pregunta no es fácil ya que no disponemos de mucha información al respecto, pero gracias a restos fosilizados de animales podemos deducir de la presencia de agentes infecciones hace ya millones de años ${ }^{2}$, mucho antes de la aparición de los primeros homínidos. Además, algunos de esos agentes han evolucionado y persisten en la actualidad convertidos en causa de zoonosis ${ }^{3}$.

La aparición de las zoonosis ha tenido siempre consecuencias negativas tanto para humanos como animales, afectando a su salud y supervivencia, provocando además graves consecuencias sociales y económicas (hambrunas, miseria, crisis social, etc.). Sin embargo, también podemos considerar aspectos positivos en las mismas tanto para humanos como animales (tratamientos farmacológicos, vacunas), cambios sociales (higiene personal y pública, hábitos alimentarios), urbanísticos (mercados, mataderos, alcantarillado, saneamiento urbano) y científicos (Inmunología, Inspección de Alimentos, Salud Pública).

Realizaremos a continuación, un recorrido histórico buscando el origen de la enfermedad en los animales y de las zoonosis. Observaremos como la necesidad de la existencia de un sanador de animales llevará a la aparición del veterinario científico. Finalmente, plantearemos cómo este nuevo profesional sanitario no se limitará a la salud animal, mediante el tratamiento y prevención de sus enfermedades, sino que se integrará en equipos multidisciplinarios sanitarios conformar lo que conocemos en la actualidad como Una Salud (en inglés ‘One Health').

\section{La enfermedad animal antes de la Veterinaria}

Ya hemos visto que la enfermedad aparece con el nacimiento de la vida animal y como los agentes que las provocan han ido evolucionando y adaptándose a las nuevas situaciones hasta llegar, en muchos casos, a afectar a la especie humana generando las zoonosis.

Existen pocos datos bibliográficos que describan explícitamente enfermedades epidémicas que hayan afectado a animales en la Antigüedad. A partir del estudio de textos clásicos algunos autores interpretan algunas de las plagas bíblicas que azotaron Egipto en tiempos de Moisés, como enfermedades epidémicas animales, así la quinta plaga podría haber sido una epidemia de peste bovina, o la sexta plaga una forma cutánea de carbunco ${ }^{4}$. En períodos posteriores, las referencias a enfermedades específicamente animales o zoonosis son escasas. Sin embargo, la preocupación por el estudio y conocimiento de dichas enfermedades se desarrolló en el mundo clásico griego y romano. Queremos destacar algunos autores del inicio y de la época de oro del conocimiento árabe los cuales recogen y amplían el conocimiento de las enfermedades infecciosas animales. Autores como Al-Razi (854-932) detalla una posible inmunización contra la viruela. El gran Avicena (980-1037) ya describe el contagio de la tuberculosis como primera enfermedad zoonótica. Finalmente, Maimónides (1135-1204), el médico de Córdoba describe la transmisión de la rabia del perro al

\footnotetext{
1 Hohenheim Research Center for Health Sciences. University of Hohenheim. Página web: https://health.unihohenheim.de/en/119312. (última fecha de consulta: 2 de junio de 2020).

${ }^{2}$ Citamos como ejemplo los recientemente descubiertos restos fosilizados de Proneusticosaurus silesiacus, un reptil marino que vivió hace 245 millones de años, descubierto en Gogolin, Silesia (Polonia) y que había padecido Tuberculosis (http://scienceinpoland.pap.pl/en/news/news\%2C29817\%2Cpolish-and-american-scientists-find-tuberculosis-prehistoricreptile.html).

${ }^{3}$ La Histiocitosis de células de Langerhans, una neoplasia osteolítica benigna, muy rara, que en la actualidad afecta principalmente a niños, ha sido descubierta en un Hadrosauro, un tipo de dinosaurio que vivió hace más de 60 millones de años en Alberta, Canadá (https://wsiltv.com/2020/02/13/rare-disease-found-in-kids-discovered-in-dinosaur-fossil/).

${ }^{4}$ Alfonso Carvajal. Las pestes bíblicas. Página web: http://elsemanaldelamancha.com/art/33107/las-pestes-biblicas (última fecha de consulta: 8 de junio de 2020).
} 
hombre mediante la saliva ${ }^{5}$. En los primeros textos específicos de medicina veterinaria, como el "Kitab albaitara" (1209) aparecen descripciones muy interesantes sobre enfermedades infecciosas y parasitarias del caballo, del camello y de otras especies de animales domésticos ${ }^{6}$. En el mundo medieval y moderno la incidencia de epidemias como las diferentes oleadas de peste bubónica llegaron a ser asimiladas por la población como grandes catástrofes naturales ante las que no había otra solución que las rogativas y la espera del fin de éstas. Además de las pérdidas directas de vidas humanas debidas a dichas enfermedades, hay que señalar las consecuencias catastróficas derivadas de las mismas en lo que se refiere a las pérdidas de cabezas de animales, con la correspondiente escasez de alimentos básicos (carne, leche, huevos, miel) y de los productos derivados de los mismos (lana, cuero, cuernos, uñas y pezuñas, etc.), esenciales para la supervivencia humana. Desde los siglos XV al XVII tenemos numerosas descripciones de sucesivas epidemias que arrasaron Europa afectando a diferentes especies animales, con enfermedades que se corresponden con las actuales rabia, carbunco, peste bovina, viruela, fiebre aftosa, sarna, cólera, etc.?. Queremos

destacar como ejemplo de ellas, la epidemia de peste bovina de 1714 que provocó la muerte de más de 1,5 millones de bovinos en toda Europa. La ausencia de especialistas en el control de dichas enfermedades llevó al papa de Roma, Clemente XI, a encargar a su médico personal el estudio de las causas de dicha epidemia, así como de las posibles medidas de control a introducir para acabar con la misma. Ahí aparece Giovanni Mario Lancisi (1654-1720) quien escribe en su obra "De Bovilla Peste" (1715) frases como "Hay una partículas extremadamente pequeñas y perniciosas que pasan de un cuerpo a otro..." y establece las primeras medidas de control de los animales para acabar con la misma: aislamiento o cuarentena de los animales enfermos, examen ante- y post-mortem de los afectados, sacrificio de aquellos animales relacionados directamente con los enfermos o muertos para evitar contagios posteriores, destrucción adecuada de restos animales contaminados (cremación, uso de sosa cáustica, etc.), prohibición de movimientos de animales con aplicación de multas (ferias, mercados, etc.) ${ }^{8}$. Por primera vez, las epidemias animales se afrontan con mediadas higiénico-sanitarias racionales.

\section{EI nacimiento de la Veterinaria}

Una de las consecuencias positivas de las epidemias mencionadas fue la necesidad de disponer de unos profesionales preparados y especializados en el estudio y contención de las enfermedades de los animales, ya fueran zoonóticas o no. De ahí surgió la creación de la primera Escuela de Medicina Veterinaria en Lion (Francia) en 1762 por parte de un ilustrado francés, Claude Bourgelat ${ }^{9}$. Desde un principio, en esta escuela y en todas las que surgirán en Europa y, posteriormente, en otros continentes, el estudio de las enfermedades animales epidémicas y transmisibles se convertirá en una prioridad para la nueva profesión veterinaria. A partir de ese momento, los nuevos profesionales veterinarios comenzarán a aplicar sus conocimientos y demostrar su eficiencia en el control de las enfermedades animales en colaboración con los otros agentes de la salud.

\section{El control de las enfermedades animales}

A la espera de los primeros veterinarios graduados en las nuevas Escuelas seguirán siendo los médicos quienes se encarguen de la lucha contra las enfermedades animales. Edward Jenner (1749-1823), un médico rural inglés, observó cómo los granjeros que tenían contacto directo con bovinos y que se contagiaban de viruela bovina por el ordeño, superaban dicha enfermedad mostrando lesiones cutáneas vesiculares, pero además resultaban posteriormente inmunes ante los brotes de viruela humana. En su obra "An Inquiry into the Causes and Effects of the variolae vaccinae" (1798) describe por primera vez la posibilidad de conseguir una inmunización de los humanos ante el agente causal de la viruela a partir de la inoculación de tejidos cutáneos lesionados generados por la enfermedad bovina ${ }^{10}$.

\footnotetext{
${ }_{5}^{5}$ Camilo Alvarez de Morales. La literatura de Al-Andalus y los animales. Página web: file://Users/David/Downloads/DialnetLaLiteraturaDeAlAndalusYLosAnimales-7427695.pdf (última fecha de consulta: 8 de junio de 2020).

${ }^{6}$ HUFF, T.E., The Rise of Early Modern Science: Islam, China, and the West (Cambridge University Press 2003) p.218. ISBN 0521529948 .

${ }^{7}$ TULLY, D.C., FARES, M.A., The tale of a modern animal plague: tracing the evolutionary history and determining the time-scale for foot and mouth disease virus [published correction appears in Virology. 2009 Apr 25; 387(1) 244]. Virology. 2008;382(2):250256. Doi: 10.1016/j.virol.2008.09.011

${ }^{8}$ MANTOVANI, A., ZANETTI, R., LANCISI, G.M., De bovilla peste and stamping out. Hist Med Vet. 18/4 (1993) 97-110

${ }^{9}$ ROBIN, D., Bourgelat et les ecoles veterinaries. Bulletin La Société d'Histoire de la Médecine et des Sciences Vétérinaires, 1/1 (2002)

10 SMITH, K.A., Edward Jenner and the small pox vaccine. Frontiers in Immunology. 2/21 (2011) 1-6. Doi:
} 
Sin embargo, esta práctica conocida posteriormente como inmunización o vacunación no era nueva. Desde el siglo X, los alquimistas taoístas chinos de la región de Szechuan, insuflaban, vía nasal, escaras de individuos que habían sufrido Viruela para provocar, en el paciente sano, una infección débil que le protegiera frente a futuras infecciones ${ }^{11}$. La insuflación se convirtió así en la primera técnica de inmunización de la historia de la salud. Esta idea se transmitió a las culturas persa y otomana las cuales, modificaron la técnica inicial: escarificaban la piel de los individuos a inmunizar, inoculando en las heridas líquido que obtenían de las lesiones vesiculares de enfermos que sufrían la enfermedad, obteniendo óptimos resultados. Gracias a un curioso personaje, Lady Montagu ${ }^{12}$ esposa de un funcionario inglés en Estambul, la variolización preventiva llegó a Inglaterra donde, gracias a sus contactos directos con la familia real inglesa, consiguió que fuera adoptada y aplicada de forma generalizada, demostrando su eficacia en la epidemia de Londres de 1721.

Una nueva enfermedad zoonótica, el ántrax o carbunco, pasó a ser identificada y controlada. La epizootia carbuncosa diezmaba el ganado de muchas comarcas francesas, en especial en los llamados "campos malditos", donde hasta un 20\% de las ovejas morían de esta infección. Casimir Joseph Davaine (1812-1882), un médico francés, descubrió al agente productor de la misma, el Bacillus anthracis, en la sangre de rumiantes muertos (1855); además, demostró que al inyectarlo en animales sanos reproducía la enfermedad (1860) ${ }^{13}$. Pero fue otro francés, en este caso un químico, Louis Pasteur (1822-1895) quien descubrió la vacuna del ántrax ${ }^{14}$. Pasteur observó, en el curso de sus investigaciones, que los animales se hacían resistentes a desarrollar el carbunco cuando se les había inyectado gérmenes de virulencia atenuada por el calor. La eficacia de la vacuna de Pasteur contra el carbunco fue demostrada en los famosos experimentos de Pouilly-le-Fort, donde el 28 de abril de 1881 inyectó su nueva vacuna a 24 ovejas y repitió la inyección tres semanas después. Quince días más tarde, inoculó bacilos de carbunco virulentos a ese grupo de ovejas e hizo lo mismo con un grupo control de animales no vacunados. Todas les del primer grupo no manifestaron el menor signo de enfermedad, mientras que les del segundo murieron todas.

Pero la aportación más famosa y conocida de Pasteur fue el desarrollo de la primera vacuna antirábica ${ }^{15}$. En 1885, a los nueve años, Joseph Meister fue mordido por un perro con rabia. Pasteur decidió tratar al niño con un virus de la rabia estudiado en conejos y debilitado posteriormente. El tratamiento duró 10 días con inyecciones diarias, gracias a las cuales el niño no desarrolló la enfermedad.

En paralelo a los trabajos de Pasteur en Francia otro microbiólogo, Robert Koch (1843-1910), en Alemania, desarrolló sus estudios principalmente sobre la tuberculosis, identificando al Mycobacterium tuberculosis como agente productor de la misma ${ }^{16}$. Sus trabajos abrieron el estudio de esta enfermedad tanto en el ámbito humano como en el animal y en la discusión de las vías de zoonóticas de transmisión entre especies (contacto directo con animales tuberculosos, consumo de su carne y leche, etc.). Todo ello generará uno de los aspectos más relevantes de la colaboración interprofesional frente a las zoonosis: la organización de la salud pública.

\section{La Salud Pública}

Como hemos relatado con algunos ejemplos en el apartado anterior, a lo largo del siglo XIX, especialmente en Europa, se produce un gran desarrollo de las ciencias microbiológicas y parasitológicas. Se identifican los agentes que provocaban dichas enfermedades y, sobretodo, se inicia el desarrollo de las vías terapéuticas que llevaran a su prevención y curación.

Fruto de ello será el inicio del estudio de lo que se llamará la Higiene Pública. Hasta bien entrado el siglo XIX, como consecuencia de la revolución industrial, la población se estaba concentrando en las grandes ciudades pasando a vivir en unas condiciones de hacinamiento e higiene deplorables. No existía aún pavimentación ni alcantarillado urbano adecuados lo que permitía la acumulación de desechos y desperdicios peligrosos por las calles. Las casas y palacios, por ejemplo, no disponían de letrinas que garantizasen la eliminación correcta de residuos orgánicos. Todo ello llevó a las autoridades a organizar los servicios

\footnotetext{
10.3389/fimmu.2011.00021

${ }^{11}$ BOYLSTON, A., The origins of inoculation. J.R. Soc. Med. 2012, 105, 309-313. Doi: 10.1258/jrsm.2012.12k044

${ }^{12}$ Lady Montagu and the introduction of inoculation. Página web: http://blog.wellcomelibrary.org/2016/05/lady-montagu-and-theintroduction-of-inoculation/ (última fecha de consulta: 12 de junio de 2020).

${ }^{13}$ SWIDERSKI, R.M., Anthrax: A History (McFarland 2014) ISBN 9780786481965.

14 FITZGERALD, J.G., Louis Pasteur - his contribution to anthrax, vaccination and the evolution of a principle of active immunization. Cal State J Med. 1923 Mar; 21(3): 101-103. PMCID: PMC1517523 PMID: 18738937

${ }^{15}$ KOURY, R., WARRINGTON, S.J., Rabies. In: StatPearls [Internet]. Treasure Island (FL): StatPearls Publishing; 2020 Jan. 2020 Jul 3.

${ }^{16}$ DANIEL, T.M., BATES, J.H., DOWNES, K.A., History of Tuberculosis. Book Editor(s): Barry R. Bloom Ph.D. First published: 16 May 1994 https://doi.org/10.1128/9781555818357.ch2
} 
sanitarios municipales que aseguraran la prevención y el control de las enfermedades, creando lo que se llamó "salud pública". Para ello se formaron equipos multidisciplinares que empezaron a aplicar una serie de medidas para mejorar la organización urbana: reubicando la localización de servicios que generaban desechos o emanaciones peligrosas (mercados, mataderos o cementerios) fuera del casco urbano; iniciando la pavimentación de las calles y de las vías de acceso con el objetivo de evitar emanaciones telúricas; el uso de la cal para el revestimiento de paredes interiores y exteriores (el encalado produce alcalinidad y dificulta la proliferación microbiana); la organización del servicio de recogida de basuras; la aplicación de drenajes en edificios y vías urbanas, con un alcantarillado adecuado, para alejar dichos residuos del contacto humano; facilitar la renovación del aire (aireación diaria de las habitaciones) para evitar la concentración de microrganismos en el ambiente, etc.

El aumento de la población urbana también generó la necesidad de proveer a los municipios de servicios de mercado que garantizasen el proveimiento de alimentos de origen animal y vegetal. Además, esos alimentos podían ser origen de enfermedades zoonóticas por lo que la necesidad de su control se convirtió en un apartado muy importante de la salud pública y el papel de los veterinarios va a resultar fundamental.

\section{Los veterinarios y el control de los alimentos}

Desde la creación de las Escuelas de Veterinaria a finales del s. XVIII, el número de los profesionales egresados de las mismas y su formación científica los llevarán a jugar un papel fundamental en este apartado. Veamos algunos ejemplos.

En 1840, a raíz de una epidemia de fiebre aftosa que afectó a los rumiantes de la provincia de Madrid, los veterinarios de la Real Escuela de Veterinaria de Madrid se revelan como los mejor capacitados para su control y erradicación, evitando la llegada de carnes contaminadas a los mercados de la ciudad. Como reconocimiento a su eficaz actuación el ayuntamiento madrileño creará el primer Servicio Municipal de Inspección Veterinaria para carnes y pescados del país que redactó el primer "Reglamento de carnes y reglamento interior de las casas-matadero" ${ }^{17}$. Algunos años más tarde, en 1857, una epidemia de carbunco en la provincia de Barcelona llevó a las autoridades municipales a crear un Servicio de Control de carnes similar al de Madrid ${ }^{18}$.

Para que todas las inspecciones municipales fueran desempeñadas por veterinarios especializados con un mismo criterio urgía agrupar las reglas técnicas de la inspección de los alimentos de origen animal. A los inspectores veterinarios no les interesaba el tratamiento o curación de las enfermedades, sino que se fijaban en las alteraciones patológicas para dictaminar la posibilidad del peligro que la ingestión de carnes, leches, etc.... podía ocasionar al organismo humano y, en consecuencia, retirar o no del mercado estos alimentos. Queremos destacar aquí la figura de un eminente veterinario, inspector del matadero de Xàtiva (Valencia), Juan Morcillo y Olalla $(1828-1908)^{19}$, quien dio un contenido científico a la inspección veterinaria en el matadero.

Fruto de sus años como inspector redactó una "Guía del Veterinario Inspector" (1858). En la misma incluía como debería ser la organización ideal de los mataderos, como llevar a cabo la inspección de carnes y pescados con una descripción pormenorizada de los patrones lesionales que se producen en las diferentes enfermedades animales. Además, incluía la inspección de la leche, de los huevos, los vegetales y las frutas.

La obra de Morcillo tuvo gran eco y su figura constituyó la referencia oficial para la redacción de numerosas normativas de aplicación estatal. Así, en 1859, una Real Orden regula la Inspección de carnes a cargo de Veterinarios en todos los municipios de España de más de 20.000 habitantes $^{20}$. En 1865 se regula inspección de la leche en Barcelona la cual se extenderá, por Real Orden (1867), a toda España ${ }^{21}$.

Así la "Guía del Veterinario Inspector" representa el primer tratado escrito por un veterinario que estudia científicamente las cuestiones referentes a la inspección de alimentos de origen animal, obra escrita con años de antelación sobre los textos alemanes y franceses.

Una vez más, una zoonosis forzará la inspección de alimentos y los veterinarios jugarán un papel fundamental. Se trató de la Triquinosis o Triquinelosis, enfermedad parasitaria causada por un nematodo (Trichinella spiralis) y que afectaba a los humanos por ingestión de carne de cerdo contaminada. En 1877 se

\footnotetext{
${ }^{17}$ JODRA TRILLO, J.E., Instauración y consolidación de la Inspección Veterinaria de Carnes en Madrid, en la primera mitad del siglo XIX. Tesis doctoral. Universidad Complutense de Madrid (2015)

${ }^{18}$ Ibíd.

$19 \mathrm{https} / /$ www.historiaveterinaria.org/update/biografia-morcillo-olalla-1456491338.pdf (última fecha de consulta: 9 de junio de 2020).

${ }^{20} \mathrm{https}: / /$ ddd.uab.cat/pub/revinscar/revinscar_a1896m10n1.pdf (última fecha de consulta: 9 de junio de 2020).

${ }^{21}$ Gaceta de Madrid (1867) Real orden regularizando el servicio de establecimientos de vacas y demás que con ellos tienen analogía. Reglamento (26 de agosto) 9-12
} 
produjo un brote epidémico en Villar del arzobispo (Valencia), y entre 1879 y 1881 se describieron nuevos focos en España ${ }^{22}$. La actuación de los veterinarios inspectores fue destacada y llevó a la introducción del triquinoscopio por Real Orden de 1887 como herramienta de trabajo en todos los mataderos del país para llevar a cabo la inspección de las carnes sospechosas de forma adecuada ${ }^{23}$.

También citaremos dentro de este apartado a un discípulo de Robert Koch, August Gärtner (1848-1934), quien describió por primera vez una bacteria causante de una intoxicación alimentaria. El llamado inicialmente bacilo de Gärtner (1888) fue posteriormente clasificado como Salmonella enteriditis ${ }^{24}$. Las salmonelosis representan en la actualidad casi un $40 \%$ de todos los casos de enfermedades de origen alimentario y es quizás la zoonosis más difundida del mundo. Las aves domésticas son uno de los reservorios principales de las salmonelas. Prácticamente cualquier alimento de origen animal (carnes contaminadas, el huevo, la leche, los subproductos de estos últimos...) puede ser fuente de infección para el hombre.

\section{Los veterinarios y las epidemias animales}

A lo largo del s. XIX las epidemias animales no solamente afectaban a la alimentación de los humanos sino también a su trabajo, al transporte, a las comunicaciones, a la guerra, etc. como eran las que afectaron a los équidos ${ }^{25}$. Las pérdidas económicas y sociales eran muy importantes y otra vez los veterinarios demostraron su capacidad de respuesta frente a las enfermedades animales en todo el mundo. Otro ejemplo sería la epidemia de peste bovina y de peste de los pequeños rumiantes de 1890 en África que provocó la muerte de más de 5,2 millones de bovinos al sur del Zambeze, siendo considerada la epidemia más devastadora que afectó a África en el s. XIX ${ }^{26}$. No solo murieron rumiantes domésticos sino también salvajes (búfalos, jirafas, etc.) provocando hambrunas y muerte de un tercio de la población de Etiopía y dos tercios de la población Masai de Tanzania.

Las autoridades sanitarias, tanto de las colonias como de las metrópolis, aplicaron en todos sus territorios una serie de regulaciones oficiales con el objetivo de garantizar la salud animal. Destacamos entre ellas: el control del movimiento e importación de animales, el aislamiento de poblaciones autóctonas (cuarentenas), la declaración obligatoria de las enfermedades animales, el sacrificio y la destrucción de los restos de los animales afectados o relacionados con la enfermedad, facilitar compensaciones o indemnizaciones a los ganaderos por las pérdidas producidas por las epidemias (el precedente de los actuales seguros animales), etc.

La necesidad de unificar la lucha contra las enfermedades animales a un nivel global llevó a la creación, en 1924, de la Oficina Internacional de Epizootias (OIE) mediante un acuerdo internacional entre los países más importantes del momento ${ }^{27}$. Muchos años más tarde, en 2003, la Oficina se transformó en lo que hoy conocemos como Organización Mundial de la Salud Animal ("World Organization for Animal Health") aunque sigue manteniendo su acrónimo original como OIE.

Las continuas oleadas de enfermedades epidémicas animales, muchas de ellas zoonóticas, que nos están afectando en los últimos tiempos (tuberculosis, pestes porcinas, fiebre aftosa, enfermedades producidas por priones, etc.) no sólo han afectado a las cabañas animales, sino que han supuesto y aún suponen un peligro para la salud y supervivencia humanas. Además, los costes económicos que generan son demasiado importantes para ignorarlas. Por ello, se ha hecho imprescindible la colaboración entre las profesiones sanitarias y la creación de equipos multidisciplinares que controlen tanto la salud animal como humana se ha convertido en una necesidad y una realidad.

\section{La mutlidisciplinariedad: 'One Health'}

Un último ejemplo de esta colaboración entre veterinarios, médicos, microbiólogos y autoridades

\footnotetext{
22 GUTIÉRREZ-GARCÍA, J.M., Triquinas, cerdos y salud pública veterinaria: la inclusión del mundo microscópico en la base científica de la inspección cárnica (Barcelona 1870s). Medicina e Historia. Revista de Estudios Históricos de las Ciencias de la Salud. 1 (2016): 4-16

${ }^{23}$ Ibíd.

${ }^{24}$ RAHMAN, H.S., MAHMOUD, B.M., OTHMAN, H.H., AMIN, K., A Review of History, Definition, Classification, Source, Transmission, and Pathogenesis of Salmonella: A Model for Human Infection. Journal of Zankoy Sulaimani 20 (2018) 3-4 (Part-A) Doi: $10.17656 /$ jzs. 10730

${ }^{25}$ KHERAJ, S., The Great Epizootic of 1872-73: Networks of Animal Disease in North American Urban Environments, Environmental History 23 (2018) 495-521 https://doi.org/10.1093/envhis/emy010

26 MACK, R. The great African cattle plague epidemic of the 1890's. Trop Anim Health Prod 2 (1970) $210-219$. https://doi.org/10.1007/BF02356441

${ }^{27}$ World Organisation for animal Health. Página web://www.oie.int/about-us/history/ (última fecha de consulta: 4 de junio de 2020).
} 
sanitarias lo encontramos en la epidemia de tifus que afectó a la ciudad de Barcelona en 1914. Ramón Turró y Darder (1854- 1926), un veterinario al frente del Laboratorio Municipal dirige un grupo multidisciplinar que consigue identificar el origen de la epidemia, aislar y demostrar su origen infeccioso, y la vía de transmisión gracias a la contaminación de parte de la red pública del suministro de agua potable a la ciudad ${ }^{28}$. Las medidas sanitarias correctoras introducidas por el consistorio municipal de Barcelona gracias a las instrucciones de Turró lograron erradicar la epidemia.

Desde el inicio del s. XXI se ha introducido el concepto de Una Salud ('One Health') ${ }^{29}$ al referirnos al hecho que la salud humana y la sanidad animal son interdependientes. Además, se le ha añadido un tercer elemento muy importante al que están vinculadas: los ecosistemas en los que coexisten. Por lo tanto, lo que empezó siendo un trabajo profesional individual se ha convertido en la labor en equipos multidisciplinares de veterinarios, médicos, biólogos, sanitarios, ecólogos, juristas, filósofos, animalistas, etc. que son los responsables, mediante un trabajo en equipo, de mantener la supervivencia de todas las especies de nuestro planeta.

\section{Bibliografía}

- BOYLSTON, A., The origins of inoculation. J.R. Soc. Med. 2012, 105, 309-313. Doi: 10.1258/jrsm.2012.12k044

- DANIEL, T.M., BATES, J.H., DOWNES, K.A., History of Tuberculosis. Book Editor(s): Barry R. Bloom Ph.D. First published: 16 May 1994 https://doi.org/10.1128/9781555818357.ch2

- FITZGERALD, J.G., Louis Pasteur - his contribution to anthrax, vaccination and the evolution of a principle of active immunization. Cal State J Med. 1923 Mar; 21(3): 101-103. PMCID: PMC1517523 PMID: 18738937

- FRESQUET-FEBRER, J.L., La febre tifoide a Barcelona (1877-1933). A: Roca RosellA (Coord) Cent anys de salut pública a Barcelona. Barcelona: Ajuntament de Barcelona, (1991) 117-129.

- GUTIÉRREZ-GARCÍA, J.M., Triquinas, cerdos y salud pública veterinaria: la inclusión del mundo microscópico en la base científica de la inspección cárnica (Barcelona 1870s). Medicina e Historia. Revista de Estudios Históricos de las Ciencias de la Salud. 1 (2016): 4-16.

- HUFF, T.E., The Rise of Early Modern Science: Islam, China, and the West (Cambridge University Press 2003) p.218. ISBN 0521529948.

- JODRA TRILLO, J.E., Instauración y consolidación de la Inspección Veterinaria de Carnes en Madrid, en la primera mitad del siglo XIX. Tesis doctoral. Universidad Complutense de Madrid (2015).

- KOURY, R., WARRINGTON, S.J., Rabies. In: StatPearls [Internet]. Treasure Island (FL): StatPearls Publishing; 2020 Jan. 2020 Jul 3.

- KHERAJ, S., The Great Epizootic of 1872-73: Networks of Animal Disease in North American Urban Environments, Environmental History $23 \quad$ (2018) 495-521 https://doi.org/10.1093/envhis/emy010

- MACK, R. The great African cattle plague epidemic of the 1890's. Trop Anim Health Prod 2 (1970) 210-219. https://doi.org/10.1007/BF02356441

- MANTOVANI, A., ZANETTI, R., LANCISI, G.M., De bovilla peste and stamping out. Hist Med Vet. 18/4 (1993) 97-110

- RAHMAN, H.S., MAHMOUD, B.M., OTHMAN, H.H., AMIN, K., A Review of History, Definition, Classification, Source, Transmission, and Pathogenesis of Salmonella: A Model for Human Infection. Journal of Zankoy Sulaimani 20 (2018) 3-4 (Part-A) Doi: 10.17656/jzs.10730

- ROBIN, D., Bourgelat et les ecoles veterinaries. Bulletin La Société d'Histoire de la Médecine et des Sciences Vétérinaires, 1/1 (2002)

- SMITH, K.A., Edward Jenner and the small pox vaccine. Frontiers in Immunology. 2/21 (2011) 16. Doi: 10.3389/fimmu.2011.00021

- SWIDERSKI, R.M., Anthrax: A History (McFarland 2014) ISBN 9780786481965.

- TULLY, D.C., FARES, M.A., The tale of a modern animal plague: tracing the evolutionary history and determining the time-scale for foot and mouth disease virus [published correction appears in

\footnotetext{
${ }^{28}$ FRESQUET-FEBRER, J.L., La febre tifoide a Barcelona (1877-1933). A: Roca RosellA (Coord) Cent anys de salut pública a Barcelona. Barcelona: Ajuntament de Barcelona, (1991) 117-129.

${ }^{29}$ Ibíd. 4.
} 
Virology. 2009 Apr 25; 387(1) 244]. Virology. 2008;382(2):250-256. Doi: 10.1016/j.virol.2008.09.011 